\title{
Quantitative steps in the Axer-Landau equivalence theorem
}

\author{
O. RAMARÉ (Marseille)
}

1. Introduction. The error term in the Prime Number Theorem is a central quantity in multiplicative number theory. Other error terms also appear, and for instance if we define

$$
\psi(x)=\sum_{n \leq x} \Lambda(n), \quad \tilde{\psi}(x)=\sum_{n \leq x} \Lambda(n) / n,
$$

we would like to be able to assess $\tilde{\psi}(x)-(\log x-\gamma)$. A theorem of Landau [10] which combines results of Axer and Landau [9, 1, 8] asserts that this latter goes to zero as soon as $\psi(x)-x$ does. After a cursory look, one could believe this problem to be readily solved by partial summation, but this is not the case. Going from $\tilde{\psi}(x)-(\log x-\gamma)$ to $\psi(x)-x$ is indeed mechanical, but the reverse is more difficult. As a matter of fact Diamond \& Zhang [4] exhibited a system of Beurling generalized integers where $\psi_{\mathcal{P}}(x) \sim x$ but where $\tilde{\psi}_{\mathcal{P}}(x)-\log x$ does not have a limit, with obvious notation. The Landau Theorem referred to above answers this question from a qualitative viewpoint. I addressed the question of a quantitative version of it in [12, and more completely together with D. Platt in [11], with applications to explicit estimates: the idea is to concentrate on $\psi(x)-x$ and to automatically derive estimates for $\tilde{\psi}(x)-(\log x-\gamma)$ (and for similar quantities with primes in arithmetic progressions). The results obtained are not (conjecturally) optimal but are still rather strong, both from a theoretical and numerical point of view; see below. The link with different error terms concerning $\sum_{p \leq x} 1 / p$ and the Euler products $\prod_{p \leq x}(1-z / p)$, for any complex number $z$ of modulus not more than 2, has further been investigated by Vanlalngaia [18].

We have known since Landau [10] that the error term of the Prime Number Theorem is linked with that of the summatory function $M(x)$ of

2010 Mathematics Subject Classification: 11M06, 11N56, 11N80.

Key words and phrases: Möbius function, Landau equivalence theorem.

Received 24 April 2017; revised 10 April 2018.

Published online 25 January 2019. 
the Möbius function. From now on we use the notation

$$
M(x)=\sum_{n \leq x} \mu(n), \quad m(x)=\sum_{n \leq x} \mu(n) / n .
$$

Inferring a quantitative error term (here, simply a bound) for $M(x)$ from the one of $\psi(x)-x$ has received attention. Let us mention the work [7] of Kienast, [16] of Schoenfeld and [5] of El Marraki, although the last two authors do not present their investigation in this perspective; and lately the paper [13]. The answers are up to now rather unsatisfactory.

One would also like to derive error terms for $m(x)$ from the one of $M(x)$. Following the well-known paper [1] of Axer, the question has later been addressed by Kienast using a tauberian argument and Lambert series in [6], and more precisely in [7]; it is initially this question that we set to investigate here. It is striking that, in Kienast's Satz 10 in [7], no direct derivation is given for what could be thought as a simple question, but an additional requirement concerning $\psi(x)$ has to be made.

We end this survey of previous research with two remarks. First, as noticed by Landau, inferring an error term for $M(x)$ once we have one for $m(x)$ is routine. Secondly, a path using identities has been investigated by Balazard [3] (see [2] for a French translation) and in [13].

The methods I used with Platt to go from $\psi(x)$ to $\tilde{\psi}(x)$ relied heavily on two explicit formulae that were compared; such tools are missing when one is dealing with the Möbius function. However, after analysing this proof, we discovered that similar information could be obtained from the Mellin transform directly, and this is the subject of the present work. Here is a corollary of the main theorem.

COROLlary. There exists a positive constant $c_{1}$ such that, for $x \geq 10$, with notation of (1.1), we have

$$
|m(x)| \ll \max _{x \leq y \leq 3 x} \frac{|M(y)|}{y}+\exp \left(-\frac{c_{1} \log x}{\log \log x}\right) .
$$

Our proof of this corollary seems to be of no use to infer numerical estimates, at least at the time of this writing. Indeed, the latest best bound for $1 / \zeta(s)$ is due to Trudgian [17] and would put a constant at least of size $10^{7}$ in front of $x^{-1 /(8 \log \log x)}$. When the emphasis is on this aspect, the path of identities taken in [3] and [13] remains a better choice.

Since our method is very general and we want to encompass several cases in one statement, we first define a general setting. The reader may want to keep the case of the Möbius function in mind. We start with a Dirichlet series

$$
F(s)=\sum_{n \geq 1} a_{n} / n^{s} \quad(\Re s>1)
$$


which we assume to be absolutely convergent in the half-plane $\Re s>1$. We further assume that there exist five constants $r, \ell \in \mathbb{Z}^{+}, c \in(0,1]$, $A, B \in[2, \infty)$, and a polynomial $R$ of degree at most $r-1$ such that the function $F(s)-R(s-1) /(s-1)^{r}$ extends holomorphically to the domain

$$
\Re s \geq 1-\frac{c}{\log (A+|\Im s|)}
$$

and satisfies there the estimate

$$
\left|F(s)-R(s-1) /(s-1)^{r}\right| \leq B \log ^{\ell}(A+|\Im s|) .
$$

The choice $r=0$ is allowed, and is indeed the case of the Möbius function, i.e. when $F(s)=1 / \zeta(s)$; we further check that $R=0$ and $\ell=1$ in this case. For the primes, we use $F(s)=-\zeta^{\prime}(s) / \zeta(s)$ together with $r=\ell=1$ and $R=1$. The above hypotheses imply classically that, for any non-negative integer $h \geq 0$, there exists a polynomial $P_{r, h}$ of degree at most $r+h$ such that $\sum_{n \leq x} a_{n} / n-P_{r, h}(\log x)$ goes to zero, and also a polynomial $Q$ of degree at most $r-1$ such that $\sum_{n \leq x} a_{n}-x Q(\log x)$ is bounded above in absolute value by a multiplicative constant times $x \exp (-c \sqrt{\log (A+x)} / 2)$. When $r=0$, we set $Q=0$. Here is our central result.

THEOREM 1.1. Let $x \geq 10$. Notation being as above, for any non-negative integer $h$, we have

$$
\begin{aligned}
\left|\sum_{n \leq x} \frac{a_{n} \log ^{h} n}{n}-P_{r, h}(\log x)\right| \ll & \max _{x \leq y \leq 3 x} \frac{\left|\sum_{n \leq y} a_{n}-y Q(\log y)\right|(\log y)^{h}}{y} \\
& +\exp \left(-\frac{c \log x}{7 \log (A+\log x)}\right) .
\end{aligned}
$$

When $r=0$, the polynomial $P_{r, h}$ is constant.

The main point is that the last error term above is better than the one previously known (recalled above). The reader will see in the proof that we only use the zero-free region up to height $\log x$.

Though the case $h=0$ has been much advertised, Axer already showed that proving that $\sum_{n \leq x} \mu(n)(\log n) / n$ is asymptotic to -1 is equivalent to showing that $M(x)$ is $o(x / \log x)$, an assertion that Kienast generalized to any power of $\log n$, provided a similar condition concerning $\psi$ is satisfied. The above theorem settles the problem in full generality in a quantitative manner.

We should maybe be more precise when comparing results over such a span of time. From 1900 until 1950-1960, there was a belief that the so-called "elementary" methods had a distinct (and lesser) power than "analytic" methods. As time went by, more and more results from the "analytic" world were converted to the "elementary" world, and it is now clear that there are no differences in the results obtainable. However, one setting or another (or 
a mix of both as often in sieve context) offers more efficiency to tackle a given set of problems.

If needed, the proof of Theorem 1.1 provides us with a localization in $[x,(1+\kappa) x]$ for $y$, for any positive $\kappa$. There remains the problem of converting the last error term into a power saving in $x$, and we state formally:

QUESTION. Is it true that for any function $F$ satisfying the above hypotheses, there exist positive constants $a$ and $b$ such that

$$
\left|\sum_{n \leq x} \frac{a_{n}}{n}-P_{r, 0}(\log x)\right| \ll \max _{x / a \leq y \leq a x} \frac{\left|\sum_{n \leq y} a_{n}-y Q(\log y)\right|}{y}+x^{-b} \text { ? }
$$

In the case of the von Mangoldt function or of the Möbius function, this can be turned into a conjecture which is readily shown to be true under the Riemann Hypothesis (on taking $b$ to be any positive real number below 1/2). In general, and for instance for Beurling numbers, the question is undecided.

Though Theorem 1.1 of [11] is a consequence of the above, getting a statement numerically as good as Theorem 1.2 of the same paper is difficult, if at all possible, with the method we develop here.

We can of course get similar corollaries with $\mu(n) \chi(n)$ rather than $\mu(n)$, where $\chi$ is a Dirichlet character, and also replace the Möbius function $\mu(n)$ by the Liouville function $\lambda(n)$. We can further see these functions as related to the number field $\mathbb{Q}$ and consider similar quantities but related to a different number field, or consider $L$-series associated to Hecke grossen Charakteren or to some modular form, and finally to Beurling numbers.

2. Preliminary lemmas. We first recall a handy integral Gorny inequality we proved in [14, Theorem 1.4].

LEMMA 2.1. Let $\mathcal{C}_{k}(a, b)$ be the class of functions $f$ over an interval $(a, b)$ (both $a$ and $b$ can be infinite) that are $k$-times differentiable, such that all $f^{(h)}$ with $h \in\{0, \ldots, k\}$ are in $L^{2}$ and $f^{(h)}(a)=f^{(h)}(b)=0$ for all $h \in\{0, \ldots, k-1\}$. Let $f$ be in $\mathcal{C}_{k}(a, b)$. Then, for any $h \in\{0, \ldots, k\}$,

$$
\int_{a}^{b}\left|f^{(h)}(v)\right|^{2} d v \leq\left(\int_{a}^{b}\left|f^{(k)}(v)\right|^{2} d v\right)^{h / k}\left(\int_{a}^{b}|f(v)|^{2} d v\right)^{1-h / k} .
$$

We rely on [15] for the smoothing process. We define, for any integer $m \geq 1$, a function over $[0,1]$ by

$$
f_{m}(t)=(4 t(1-t))^{m} .
$$

This function satisfies

$$
f_{m}^{(k)}(0)=f_{m}^{(k)}(1)=0 \quad(0 \leq k \leq m-1) .
$$

We recall part of [15, Lemma 6]. 
LEMMA 2.2.

$$
\begin{aligned}
& \left\|f_{m}\right\|_{1}=\frac{2^{2 m} m !^{2}}{(2 m+1) !}, \quad\left\|f_{m}^{(m)}\right\|_{2}=\frac{2^{2 m} m !}{\sqrt{2 m+1}}, \\
& \left\|f_{m}^{(k)}\right\|_{2} \ll \sqrt{m}(4 m / e)^{k} \quad(k \leq m) .
\end{aligned}
$$

Proof. The first two statements come from [15, Lemma 6], and we deduce the last one by appealing to Lemma 2.1.

$$
\left\|f_{m}^{(k)}\right\|_{2} \leq\left(2^{2 m} \frac{m !}{\sqrt{2 m+1}}\right)^{k / m}\left(\frac{2^{4 m}(2 m) !^{2}}{(4 m+1) !}\right)^{(m-k) /(2 m)}
$$

since $\left\|f_{m}\right\|_{2}=\sqrt{\left\|f_{2 m}\right\|_{1}}$. Hence, on using Lemma 2.1.

$$
\begin{aligned}
\left\|f_{m}^{(k)}\right\|_{2} /\left\|f_{m}\right\|_{1} & \leq\left(\frac{m !^{2}}{(2 m+1)} \frac{(4 m+1) !}{(2 m) !^{2}}\right)^{k /(2 m)} \frac{(2 m) !(2 m+1) !}{\sqrt{(4 m+1) ! m !^{2}}} \\
& \ll\left(\frac{m^{2 m}}{e^{2 m}} \frac{(4 m)^{4 m} e^{4 m}}{e^{4 m}(2 m)^{4 m}}\right)^{k /(2 m)} \frac{(2 m / e)^{4 m} m^{2}}{m^{5 / 2}(4 m / e)^{2 m}(m / e)^{2 m}} \\
& \ll \sqrt{m}(4 m / e)^{k}
\end{aligned}
$$

as claimed.

Lemma 2.3. When $u>1$ and $k \geq 0$, we have

$$
\frac{d^{k}}{d u^{k}}\left(\frac{1}{(\log u)^{2}}\right)=(-1)^{k} \sum_{2 \leq j \leq k+2} \frac{c_{k}(j)}{j ! u^{k}(\log u)^{j}}
$$

for some non-negative coefficients $c_{k}(j)$ that satisfy $\sum_{j} c_{k}(j)=k !$.

Proof. We prove the existence of the coefficients $c_{k}(j)$ by recursion:

$$
\left(\frac{1}{(\log u)^{2}}\right)^{(k+1)}=(-1)^{k+1} \sum_{2 \leq j \leq k+2}\left(\frac{k c_{k}(j)}{j ! u^{k+1}(\log u)^{j}}+\frac{j c_{k}(j)}{j ! u^{k+1}(\log u)^{j+1}}\right)
$$

and thus $c_{k+1}(j)=k c_{k}(j)+c_{k}(j-1)$ with $c_{k}(1)=c_{k}(k+3)=0$. Define

$$
P_{k}(X)=\sum_{2 \leq j \leq k+2} c_{k}(j) X^{j} .
$$

The recursion gives

$$
P_{k+1}(X)=(X+k) P_{k}(X), \quad P_{0}(X)=X^{2},
$$

whence $P_{k}(X)=(X+k-1)(X+k-2) \cdots X^{2}$. The Taylor expansion of $P_{k}(X) / X$ is given by the (unsigned) Stirling numbers of the first kind $|s(k, j)|$, but we do not need this. It is enough to see that

$$
P_{k}(1)=k !=\sum_{j} c_{k}(j) \text {. }
$$

This does not lose much since $c_{k}(k+2)=(k-1)$ !. 
From $f_{m}$, we define (almost as in [11, Section 4])

$$
g_{m}(t)= \begin{cases}1 & \text { when } 0<t \leq 2 \\ 1-\left\|f_{m}\right\|_{1}^{-1} \int_{0}^{t-2} f_{m}(u) d u & \text { when } 2 \leq t \leq 3 \\ 0 & \text { when } t \geq 3\end{cases}
$$

Note that $0 \leq g_{m}(t) \leq 1$.

We consider, for $\Re s \leq 1$, the Mellin transform

$$
H_{m}(s)=\int_{2}^{\infty} \frac{1-g_{m}(u)}{u(\log u)^{2}} u^{s-1} d u .
$$

We need the denominator $(\log u)^{2}$ at infinity, but since this function vanishes at $u=1$, we have shifted the smoothing from 2 onwards.

Lemma 2.4. When $\Re s \leq 1$, we have $\left|H_{m}(s)\right| \ll_{m} 1$. The function $H_{m}$ extends to a holomorphic function in the half-plane $\Re s \leq 1$. When $|\Im s| \geq 1$, we have, uniformly in $m$,

$$
\left|H_{m}(s)\right| \ll 1 /|s|, \quad\left|H_{m}(s)\right| \ll(2 m)^{m} /|s|^{m+1} .
$$

Proof. We define $1-g_{m}(u)=a_{m}(u)$ so that, by Lemma 2.2 when $1 \leq$ $k \leq m+1$, and directly otherwise, we have $a_{m}^{(k)}(u) \ll(2 m / e)^{k}$ for $k \in$ $\{0, \ldots, m+1\}$. We further set $a_{m}(u) /(\log u)^{2}=b_{m}(u)$. Notice that, for any $k \in\{0, \ldots, m\}$, we have $b_{m}^{(k)}(2)=0$. Hence we can use $k \in\{0, \ldots, m+1\}$ integrations by parts to reach

$$
H_{m}(s)=\frac{(-1)^{k}}{(s-1) s \cdots(s-1+k)} \int_{2}^{\infty} b_{m}^{(k)}(u) u^{s-2+k} d u .
$$

We use this expression for $k=m+1$. Leibniz's formula together with Lemma 2.3 gives

$$
\begin{aligned}
b_{m}^{(m+1)}(u) & =\sum_{0 \leq k \leq m+1}\left(\begin{array}{c}
m+1 \\
k
\end{array}\right) a_{m}^{(k)}(u) \sum_{2 \leq j \leq 2+m+1-k} \frac{c_{m+1-k}(j)}{(m+1-k) ! u^{m+1-k}(\log u)^{j}} . \\
= & \frac{a_{m}(u)}{u^{m+1}(m+1) !} \sum_{2 \leq j \leq m+3} \frac{c_{m+1}(j)}{(\log u)^{j}} \\
& +\sum_{1 \leq k \leq m+1}\left(\begin{array}{c}
m+1 \\
k
\end{array}\right) a_{m}^{(k)}(u) \sum_{2 \leq j \leq m+3-k} \frac{c_{m+1-k}(j)}{(m+1-k) ! u^{m+1-k}(\log u)^{j}} .
\end{aligned}
$$

The second part of this expression vanishes when $u \geq 3$ and is otherwise 
bounded above by

$$
\begin{aligned}
& \ll \sum_{1 \leq k \leq m+1}\left(\begin{array}{c}
m+1 \\
k
\end{array}\right) m^{k} \frac{(m+1-k) !}{(\log 2)^{m+3-k}} \\
& \ll \frac{(m+1) ! \kappa}{(\log 2)^{m}} \sum_{0 \leq k \leq m+1} \frac{m^{k}(\log 2)^{k}}{k !} \\
& \ll \frac{(m+1) !}{(\log 2)^{m}} e^{m \log 2} \ll m^{3 / 2}\left(\frac{2 m}{e \log 2}\right)^{m} \ll(2 m)^{m} .
\end{aligned}
$$

Therefore, when $|\Im s| \geq 1$, we get

$$
H_{m}(s) \ll \frac{1}{|s|^{m+1}}\left(\int_{2}^{\infty} \frac{u^{\Re s-2+m+1} d u}{u^{m+1}(\log u)^{2}}+(2 m)^{m}\right) \ll(2 m)^{m} /|s|^{m+1}
$$

as required.

3. The decomposition. To ease typographical work, we define

$$
S_{h}(x)=\sum_{n \leq x} a_{n}, \quad \tilde{S}_{h}(x)=\sum_{n \leq x} a_{n}(\log n)^{h} / n .
$$

It will also be helpful to use the shortcut

$$
\rho_{h}(t)=(\log t)^{h} / t .
$$

We first use integration by parts to remove the $(\log n)^{h} / n$ :

$$
\begin{aligned}
\tilde{S}_{h}(x)= & \frac{S(x)(\log x)^{h}}{x}-\int_{1}^{x} S(t) \rho_{h}^{\prime}(t) d t \\
= & \frac{(S(x)-x Q(\log x))(\log x)^{h}}{x}+Q(\log x)(\log x)^{h} \\
& -\int_{1}^{x} Q(\log t) t \rho_{h}^{\prime}(t) d t-\int_{1}^{\infty}(S(t)-t Q(\log t)) \rho_{h}^{\prime}(t) d t \\
& +\int_{x}^{\infty}(S(t)-t Q(\log t)) \rho_{h}^{\prime}(t) d t
\end{aligned}
$$

since our hypotheses ensure that $S(t)-t Q(\log t)=\mathcal{O}\left(t / \log (2 t)^{h+2}\right)$. Though much more is true, such an estimate is enough to prove that the integral $\int_{1}^{\infty}(S(t)-t Q(\log t)) \rho_{h}^{\prime}(t) d t$ is absolutely convergent. By unicity, we have

$$
\begin{aligned}
P_{r, h}(\log x)= & Q(\log x)(\log t)^{h}+\int_{1}^{x} Q(\log t) t \rho_{h}^{\prime}(t) d t \\
& +\int_{1}^{\infty}(S(t)-t Q(\log t)) \rho_{h}^{\prime}(t) d t .
\end{aligned}
$$


At this level, we introduce new notation:

$$
\Delta(x)=\sum_{n \leq x} a_{n}-x Q(\log x), \quad \tilde{\Delta}_{h}(x)=\sum_{n \leq x} \frac{a_{n}(\log n)^{h}}{n}-P_{r, h}(\log x) .
$$

We will use the decomposition

$$
\begin{aligned}
\tilde{\Delta}_{h}(x)= & \frac{\Delta(x)(\log x)^{h}}{x}-\int_{x}^{3 x} \Delta(t) g_{m}(t / x) \rho_{h}^{\prime}(t) d t \\
& -\int_{x}^{\infty} \Delta(t)\left(1-g_{m}(t / x)\right) \rho_{h}^{\prime}(t) d t .
\end{aligned}
$$

Our next task is to express the last summand in terms of Mellin transforms. On recalling (2.6), the Mellin inversion formula gives, when $u>0$,

$$
\frac{1-g_{m}(u)}{u(\log u)^{2}}=\frac{1}{2 i \pi} \int_{1-i \infty}^{1+i \infty} H_{m}(s) u^{-s} d s
$$

and thus

$$
\begin{aligned}
\int_{x}^{\infty} \Delta(t)\left(1-g_{m}(t / x)\right) \rho_{h}^{\prime}(t) d t & =\int_{1}^{\infty} \Delta(t)\left(1-g_{m}(t / x)\right) \rho_{h}^{\prime}(t) d t \\
& =\frac{1}{2 i \pi} \int_{1-i \infty}^{1+i \infty} H_{m}(s) x^{s} \int_{1}^{\infty} \Delta(t) t(\log t)^{2} \rho_{h}^{\prime}(t) \frac{d t}{x t^{s}} d s
\end{aligned}
$$

At this level we employ the exact expression of $\rho_{h}^{\prime}$, i.e. $t^{2} \rho_{t}^{\prime}(t)=-(\log t)^{h}+$ $h(\log t)^{h-1}$. We note that when $\Re s>1$, we have

$$
F(s) / s=\int_{1}^{\infty} S(t) d t / t^{s+1}
$$

where the integral converges absolutely. As a consequence, for any nonnegative integer $k$, the following holds:

$$
\frac{d^{k}}{d s^{k}}(F(s) / s)=(-1)^{k} \int_{1}^{\infty} S(t)(\log t)^{k} d t / t^{s+1} .
$$

We infer from this formula that

$$
(-1)^{h+1} \int_{0}^{\infty} S(t) t(\log t)^{2} \rho_{h}^{\prime}(t) \frac{d t}{t^{s}}=\frac{d^{h+2}}{d s^{h+2}}(F(s) / s)+h \frac{d^{h+1}}{d s^{h+1}}(F(s) / s) .
$$

We define

$$
\begin{aligned}
G_{h}(s)= & (-1)^{h} \frac{d^{h+2}}{d s^{h+2}}(F(s) / s) \\
& +(-1)^{h} h \frac{d^{h+1}}{d s^{h+1}}(F(s) / s)-\frac{R_{h}(s-1)}{(s-1)^{h+r+2}}
\end{aligned}
$$


where $R_{h}$ is a polynomial of degree at most $h+r+1$ and moreover $R_{h}(s-1) /(s-1)^{h+r+2}$ is the polar part of $G_{h}(s)$ at $s=1$. The final thing to notice is that

$$
\int_{1}^{\infty} \Delta(t) t(\log t)^{2} \rho_{h}^{\prime}(t) \frac{d t}{t^{s}} d s=G_{h}(s)
$$

for $\Re s \geq 1$. This is however obvious when $\Re s>1$ by following the above reasoning, on simply replacing (3.6) by

$$
\left(F(s)-\frac{R_{h}(s-1)}{(s-1)^{r}}\right) / s=\int_{1}^{\infty}(S(t)-t Q(\log t)) \frac{d t}{t^{s+1}} .
$$

The extension to $\Re s \geq 1$ follows by unicity of analytic continuation, say. Here is the final result of this section:

$$
\begin{aligned}
\tilde{\Delta}_{h}(x)= & \frac{\Delta(x)(\log x)^{h}}{x}+\int_{x}^{3 x} \Delta(t) g_{m}(t / x) \rho_{h}^{\prime}(t) d t \\
& -\frac{1}{2 i \pi} \int_{1-i \infty}^{1+i \infty} H_{m}(s) x^{s-1} G_{h}(s) \frac{d s}{s} .
\end{aligned}
$$

4. Proof of Theorem 1.1. In order to use formula (3.12), we need some bounds for $G_{h}$. We take $s$ in the region

$$
\Re s \geq 1-\frac{c}{2 \log (A+|\Im s|)} .
$$

Then, to bound $G_{h}(s)$, we use Cauchy's Theorem on a circle centered at $s$ and of radius $(c / 2) / \log (A+|\Im s|)$, immediately deducing that the modulus of $G_{h}(s)$ is bounded by a constant multiple of $\log ^{\ell+h+2}(A+|\Im s|)$ in the region defined by (4.1).

Once this is established, we select a parameter $T \geq 2$ and shift the line of integration to

$$
\Re s=1-\frac{c}{2 \log (A+T)}
$$

when $|\Im s| \leq T$. When $|\Im s| \geq T$, we shift the line of integration to $\Re s=1$, and we complete this path with the two horizontal segments $|\Im s|=T$ and $1-c / \log (A+T) \leq \Re s \leq 1$. On this line $\mathcal{L}$, we get

$$
\begin{aligned}
\int_{\mathcal{L}} \mid H_{m}(s) & x^{s-1} G_{h}(s) \frac{d s}{s} \mid \\
& \ll\left(x^{-(c / 2) / \log (A+T)} \log T+\frac{1}{T^{2}}\left(\frac{2 m}{T}\right)^{m}\right)(\log (A+T))^{\ell+h+2} .
\end{aligned}
$$


We select

$$
T=4 m, \quad m=[\log x] .
$$

When $x$ is so large that

$$
\frac{c}{3} \log x \geq(\ell+h+2)(\log \log (A+4 \log x))^{2},
$$

say $x \geq x_{0}(A, \ell+h, c)$, we have

$$
\int_{\mathcal{L}}\left|H_{m}(s, \kappa) x^{s-1} G_{h}(s) \frac{d s}{s}\right| \ll_{\kappa} \exp \left(-\frac{c \log x}{7 \log (A+\log x)}\right) .
$$

When $x$ is smaller than $x_{0}(A, \ell+h, c)$, we adjust the constant in the above inequality; indeed the left hand side is bounded is this range. This completes the proof of Theorem 1.1 .

\section{References}

[1] A. Axer, Beitrag zur Kenntnis der zahlentheoretischen Funktionen $\mu(n)$ und $\lambda(n)$, Prace Mat.-Fiz. 21 (1910), 65-95.

[2] M. Balazard, Remarques élémentaires sur la fonction de Moebius, private communication (2011), hal-00732694.

[3] M. Balazard, Elementary remarks on Möbius' function, Proc. Steklov Inst. Math. 276 (2012), 33-39.

[4] H. G. Diamond and W.-B. Zhang, A PNT equivalence for Beurling numbers, Funct. Approx. Comment. Math. 46 (2012), 225-234.

[5] M. El Marraki, Fonction sommatoire de la fonction $\mu$ de Möbius, majorations asymptotiques effectives fortes, J. Théor. Nombres Bordeaux 7 (1995), 407-433.

[6] A. Kienast, Extension to other series of Abel's and Tauber's theorems on power series, Proc. London Math. Soc. 25 (1926), 45-52.

[7] A. Kienast, Über die Äquivalenz zweier Ergebnisse der analytischen Zahlentheorie, Math. Ann. 95 (1926), 427-445.

[8] E. Landau, Über die Äquivalenz zweier Hauptsätze der analytischen Zahlentheorie, Sitzungsber. Akad. Wiss. Wien Math.-Nat. Kl. IIa 120 (1911), 973-988.

[9] E. Landau, Neuer Beweis der Gleichung $\sum_{k=1}^{\infty} \frac{\mu(k)}{k}=0$, Berlin, 1899, 16 pp.

[10] E. Landau, Über einige neuere Grenzwertsätze, Rend. Circ. Mat. Palermo 34 (1912), 121-131.

[11] D. J. Platt and O. Ramaré, Explicit estimates: from $\Lambda(n)$ in arithmetic progressions to $\Lambda(n) / n$, Experiment. Math. 26 (2017), 77-92.

[12] O. Ramaré, Explicit estimates for the summatory function of $\Lambda(n) / n$ from the one of $\Lambda(n)$, Acta Arith. 159 (2013), 113-122.

[13] O. Ramaré, Explicit estimates on several summatory functions involving the Moebius function, Math. Comp. 84 (2015), 1359-1387.

[14] O. Ramaré, Modified truncated Perron formulae, Ann. Blaise Pascal 23 (2016), 109128.

[15] O. Ramaré and Y. Saouter, Short effective intervals containing primes, J. Number Theory 98 (2003), 10-33.

[16] L. Schoenfeld, An improved estimate for the summatory function of the Möbius function, Acta Arith. 15 (1969), 223-233. 
[17] T. Trudgian, Explicit bounds on the logarithmic derivative and the reciprocal of the Riemann zeta-function, Funct. Approx. Comment. Math. 52 (2015), 253-261.

[18] R. Vanlalngaia, Explicit Mertens sums, Integers 17 (2017), A11, 18 pp.

O. Ramaré

CNRS / Institut de Mathématiques de Marseille

Aix Marseille Université, U.M.R. 7373

Site Sud, Campus de Luminy, Case 907

13288 Marseille Cedex 9, France

E-mail: olivier.ramare@univ-amu.fr 
